\title{
¿Qué valoraciones tiene el alumnado de pedagogía sobre los artículos de investigación?
}

What valuations have the student body of pedagogy about research articles?

\author{
Volumen 19, Número 3 \\ Setiembre-Diciembre \\ pp. $1-23$ \\ Haylen Perines \\ Rodrigo Vega
}

Citar este documento según modelo APA

Perines, Haylen y Vega, Rodrigo. (2019). ¿Qué valoraciones tiene el alumnado de pedagogía sobre los artículos de investigación?. Revista Actualidades Investigativas en Educación, 19(3), 1-23. Doi. 10.15517/aie.v19i3.38640 


\section{¿Qué valoraciones tiene el alumnado de pedagogía sobre los artículos de investigación? \\ What valuations have the student body of pedagogy about research articles?}

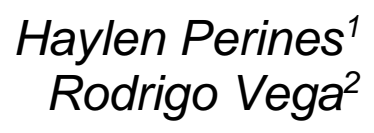

Resumen: La investigación educativa en la formación de profesorado es un elemento fundamental para su óptima preparación profesional. Gracias a la investigación, el profesorado del futuro puede conocer saberes actualizados sobre su propia práctica y contar con una base para formular y justificar sus puntos de vista. Bajo esta lógica, los objetivos de este artículo son: conocer las valoraciones que el alumnado de pedagogía tiene de los artículos de investigación y conocer las visiones del alumnado de pedagogía sobre la vinculación entre los artículos de investigación y elementos de su futura práctica docente. Para lograr estos propósitos se opta por un enfoque cualitativo en el cual se utilizaron entrevistas semi - estructuradas y grupos focales con 59 estudiantes de la Universidad de La Serena (Chile) que cursaban tercer año y que pertenecían a distintas carreras de pedagogía. La investigación se realizó entre abril y julio del año 2018. Los resultados indican que las personas que participaron en el estudio valoran positivamente los artículos de investigación, reconocen el potencial que poseen para su formación y también su importancia para la toma de decisiones en su futura práctica docente. Entre las conclusiones se hace un llamado a las personas formadoras de docentes, a las administraciones de las universidades, a personal investigador educativo y a las personas encargadas del área de investigación de las universidades para que tomen conciencia sobre la importancia de la formación en investigación del profesorado del futuro y de la inclusión oportuna de los artículos de investigación en su preparación pedagógica.

Palabras clave: investigación, docente, formación profesional, estudiantado universitario.

Abstract: Educational research in the training of teachers is a fundamental element for their optimal professional preparation. Thanks to the research, future teachers can know updated knowledge about their own practice and have a basis to formulate and justify their points of view. This research seeks to know the valuations that pedagogy students have of research articles as a resource in their training and know the visions that students have about the link between research articles and elements of their future teaching practice. To achieve these purposes, a qualitative approach is chosen in which semi - structured interviews and focus groups are used with 59 students from the University of La Serena (Chile) who are in their third year and who belong to different careers. The research was done between April and July 2018. The results indicate that the people who participated in the study positively value the research articles, recognize the potential they possess for their training and their importance for decision making in their future teaching practice. Among the conclusions, a call is made to teachers' trainers, to university administrations, to educational researchers and researchers already in charge of the research area of the universities, so that they become aware of the importance of the research training of the teachers of tomorrow and of the timely inclusion of research articles in their pedagogical preparation.

Keywords: research, teacher, vocational training, college student.

\footnotetext{
${ }_{1}$ Docente de la Universidad de La Serena, Chile. Dirección electrónica: haylen.perines@userena.cl

2 Docente de la Universidad de La Serena, Chile. Dirección electrónica: ravega@userena.cl
}

Artículo recibido: 5 de marzo, 2019

Enviado a corrección: 30 de mayo, 2019

Aprobado: 5 de agosto, 2019 


\section{Introducción}

"Ganamos muchos conocimientos importantes haciendo investigaciones educativas a pequeña escala. Aprendimos cómo llevar a cabo una investigación educativa. Además de esto, aprendimos cómo investigar y preparar un cuestionario". Esta cita, extraída del trabajo de Demircioglu (2008, p. 10), hace referencia a las valoraciones que el estudiantado de pedagogía tiene sobre un curso en investigación al que han asistido. Queda claro que son percepciones positivas. Sin embargo, llama la atención que, en el mismo estudio, el alumnado señala que su preparación en investigación ha sido prácticamente inexistente: “(...) son cosas nuevas para mí, antes apenas habíamos hablado de investigación" (Demircioglu, 2008, p. 13).

Estas evidencias ratifican lo que otros estudios ya han identificado: la investigación educativa en la formación de profesorado es un elemento fundamental para su óptima preparación profesional (Elliott, 2001; Grossman y McDonald, 2008; Perines y Murillo, 2017a; Slavin, 2002). Gracias a la investigación, el profesorado del futuro puede conocer saberes actualizados sobre su propia práctica y contar con una base para formular y justificar sus puntos de vista (Counsell, Evans, McIntyre y Raffan, 2000).

Esta temática ha posibilitado el surgimiento de una línea de investigación que aborda las valoraciones de docentes en formación sobre la investigación educativa (Alhumidi y Uba, 2017; Altrichter y Mayr, 2004; Damşa, 2018; Demircioglu, 2008; Gitlin, Barlow, Burbank, Kauchak, y Stevens, 1999; Pendry y Husbands, 2000; Perines y Murillo, 2017b; Pring, 2017). Si bien es una arista investigativa que va en crecimiento, en ella no existen abundantes evidencias que indaguen en las valoraciones que el personal docente, en formación, tiene específicamente sobre los artículos de investigación. Indagar en la lectura de los artículos de investigación por parte del profesorado del mañana todavía es un tema en desarrollo y es a lo que apunta, precisamente, este artículo de investigación.

\section{Referente teórico}

Lograr cambios en la relación entre la investigación educativa y la práctica docente requiere trabajar con docentes en formación y hacer modificaciones concretas en su preparación profesional. Un buen punto de partida es la inclusión temprana de la investigación educativa en los programas de formación de profesorado (Pring, 2017). En esta línea, Alhumidi y Uba (2017) mencionan la imperiosa necesidad de "alfabetizar" al 
profesorado en los fundamentos de la investigación, lo que mejoraría su interés por leer y escribir investigación.

Desde la perspectiva de Forner (2000), la relación entre la investigación y la formación del profesorado se debe optimizar a través de tres elementos:

1. La investigación en la formación: se refiere al incremento de la presentación y análisis de estudios e investigaciones como recurso en las materias curriculares.

2. La investigación para la formación: se refiere al uso de la investigación sobre contenidos curriculares por parte del alumnado, como una opción de mejora de los procesos de enseñanza-aprendizaje y como alternativa de las rutinas tradicionales del profesorado.

3. La formación para la investigación: se refiere a la capacitación del estudiantado en materias de investigación para que puedan colaborar y desarrollar proyectos de investigación vinculados a su práctica profesional.

Para desarrollar estos tres aspectos, Forner (2000) destaca que se deben excluir radicalmente aquellas viejas oraciones como:

Se trata de estudios de primer ciclo o de diplomatura y por lo tanto no tienen por qué investigar", "los maestros ya tienen bastante trabajo y obligaciones para que encima ahora tengan que hacer investigaciones", "tienen muy poca preparación y les falta tiempo". Este tipo de discursos solo ratifican la desconfianza hacia la capacidad intelectual del colectivo docente y "la negación de su emancipación e independencia profesional. (Forner, 2000, p. 9)

El profesorado del mañana debería ser capaz de encontrar, reflexionar críticamente y aplicar literatura de investigación para su contexto específico (Altrichter y Mayr, 2004). Si el alumnado de las pedagogías reconoce el valor que tienen las evidencias de la investigación para la práctica educativa, se asume que esto tiene una influencia favorable en su motivación para ejercer la pedagogía y en su rendimiento como profesionales informados y "alfabetizados" en investigación educativa (Eccles y Wigfield, 2002; Vetter e Ingrisani, 2013). Respecto a los estudios empíricos que indagan en las valoraciones sobre la investigación educativa de docentes en formación, existen opiniones críticas sobre dicha formación y también algunas valoraciones más positivas acerca de ella (Damşa, 2018; Demircioglu, 
2008; Gitlin et al., 1999; Jyrhämä et al., 2008; Kutlay, 2013; MacDonald, Badger y White, 2001; Pendry y Husbands, 2000; Perines y Murillo, 2017b; Pring, 2017).

En el primer grupo, vemos el trabajo de Gitlin et al. (1999) en cuyos resultados se menciona que el profesorado en formación solo conoce la investigación de manera superficial y que saben muy poco sobre el trabajo que llevan a cabo los investigadores e investigadoras. En otros estudios (Demircioglu, 2008; MacDonald et al., 2001), se le da especial énfasis al análisis crítico de los programas de formación de profesorado, y se alude a la importancia de remediar las debilidades de los planes de estudio en materias de investigación.

En el otro lado están las investigaciones que aportan valoraciones positivas (Damşa, 2018; Demircioglu, 2008; Jyrhämä et al., 2008; Perines y Murillo, 2017b). Quienes participan en el estudio de Jyrhämä et al. (2008) valoran que, en el curso de investigación al que asistieron, pudieron aprender sobre los fundamentos de la investigación educativa. Resultados similares obtienen Perines y Murillo (2017b), cuyas personas participantes creen que conocer la investigación es un saber fundamental para su futuro trabajo en el aula. Sin embargo, esperarían tener un contacto más temprano con ella desde los primeros años de estudio.

En esta misma línea, Haberfellner y Fenzl (2017) mencionan que la mayoría de las personas participantes reconocen la utilidad de la investigación educativa y creen que en su formación es vital adquirir conocimientos sobre ella para mejorar sus habilidades. Por su parte, los resultados del estudio de Damşa (2018) muestran que las actividades de formación en investigación, que posibilitan una conexión con la práctica, generan que estudiantes de pedagogía construyan experiencias significativas para su formación pedagógica.

Considerando los propósitos de este estudio es necesario mencionar las investigaciones que hayan usado la lectura de artículos de investigación como parte de su metodología (p. ej., Bartels, 2003; Murillo, Perines y Lomba, 2017; Pendry y Husbands, 2000; Zeuli, 1994). Según un orden cronológico, vemos que Zeuli (1994), quien puede ser considerado como pionero en usar esta metodología, les solicitó a 13 docentes en ejercicio que leyeran tres artículos de investigación educativa. En sus resultados queda evidenciado que los artículos de investigación son más creíbles para el profesorado si aportan ejemplos de casos similares a sus propios contextos educativos. Seis años después, Pendry y Husbands (2000) indagaron en los aportes que los artículos de investigación pueden 
significar para un grupo de docentes de secundaria que cursan un posgrado. Los hallazgos de este estudio dan cuenta de una visión positiva hacia el valor de los artículos para su desarrollo profesional, pero las personas participantes asumen que en la realidad no leen artículos de investigación con frecuencia. En el caso de Bartels (2003), la población participante de la investigación fueron tres docentes y tres personas dedicadas a la investigación, a quienes el autor solicita leer un artículo de difusión y otro académico. Los resultados reflejan que el profesorado asocia la credibilidad de un artículo a la posibilidad de conexión con el aula y, si el artículo no les proporciona dicha conexión, lo consideran un texto poco relevante. Con la intención de replicar algunas de las ideas de Zeuli (1994) y Bartels (2003), Murillo, Perines y Lomba (2017) solicitan a veinte docentes en ejercicio que lean dos tipos de artículos de investigación educativa: uno más académico y otro de difusión. Quienes participan en este estudio mencionan que los artículos son importantes para su carrera profesional, pero son críticos con la falta de utilidad de los resultados y con el lenguaje especializado que estos utilizan.

\section{Metodología}

Este apartado se organiza de la siguiente forma: primero, se mencionan los objetivos y el enfoque metodológico; posteriormente, se especifican las personas participantes, las técnicas de recolección de datos y la forma en que se analizaron los datos recopilados.

Los objetivos de este artículo son los siguientes:

- Conocer las valoraciones que el alumnado de pedagogía tiene de los artículos de investigación como un recurso pedagógico en su formación.

- Conocer las visiones del alumnado de pedagogía sobre la vinculación entre los artículos de investigación y elementos de su futura práctica docente.

\subsection{Enfoque}

Se utilizó el enfoque cualitativo, decisión que se justifica en la naturaleza comprensiva y descriptiva que caracteriza las intenciones del estudio (Corbin y Strauss, 2008). El paradigma que sustenta la investigación es interpretativo, lo que implica determinar los significados de las acciones humanas y de la vida social a través del análisis de una realidad dinámica y múltiple (Gil Álvarez, León González y Morales Cruz, 2017). De esta manera, se opta por el enfoque cualitativo al pretender describir y comprender las valoraciones que el 
estudiantado de pedagogía de la Universidad de La Serena tiene sobre los artículos de investigación.

\subsection{Participantes}

Las personas participantes del estudio, seleccionadas mediante un criterio de conveniencia, son dos grupos de estudiantes (41 y 18 participantes respetivamente) de la Universidad de La Serena (Chile) que cursan tercer año y que pertenecen a distintas carreras de pedagogía. Se opta por estudiantes de tercer nivel porque, al estar prácticamente en la mitad de su carrera, pueden tener una opinión fundamentada sobre elementos pertinentes a su formación. Además, se buscó explícitamente que fueran estudiantes pertenecientes a distintas carreras de pedagogía, para así contar con una diversidad de valoraciones y opiniones (la tabla 1 muestra las carreras a las cuales pertenece el grupo de estudiantes). Cabe mencionar que el estudiantado fue informado previamente sobre los objetivos del estudio y firmaron voluntariamente un consentimiento informado que detalla las características y que compromete, por parte de quienes llevan a cabo la investigación, la confidencialidad de los datos proporcionados.

Tabla 1

Participantes del estudio por carrera, Universidad de La Serena, Chile, 2018

\begin{tabular}{l|l}
\hline Carrera & $\mathrm{N}^{\circ}$ de estudiantes \\
\hline $\begin{array}{l}\text { Pedagogía en inglés, educación diferencial } \\
\text { y castellano y filosofía }\end{array}$ & 41 \\
\hline Pedagogía en biología y química, música & 18 \\
\hline \multicolumn{2}{l}{ Fuente: Elaboración propia (2019). }
\end{tabular}

\subsection{Técnicas de recolección}

Las técnicas de recolección de datos fueron la entrevista semiestructurada y los grupos focales.

La revisión de la literatura permitió determinar las principales categorías o ejes temáticos de la investigación sobre los cuales fue posible planificar la recopilación de información en ambas técnicas. Estos ejes temáticos se convertirían en las categorías a priori a la entrada al campo. Este razonamiento inductivo asume que, en el transcurso del estudio, y como productos de los datos recabados, emergen nuevas categorías de análisis identificadas a posteriori. Las categorías que se establecieron a priori son las siguientes: 
- Interés por la lectura de artículos.

- Valoración de las experiencias vividas con artículos de investigación.

- Relación entre la información de los artículos y los elementos de la futura práctica docente.

Por otra parte, las categorías que surgieron a posteriori a la entrada al campo son:

- Visión crítica de la formación en investigación educativa.

- Valoración de ciertas temáticas presentes en los artículos: bullying, ciberbullying, atención a la diversidad, entre otras.

- La investigación educativa como una herramienta de actualización de conocimientos.

A ambos grupos de estudiantes se les hizo entrega, en formato impreso, de nueve artículos de investigación educativa previamente seleccionados. La selección de los textos se basó en los siguientes criterios:

1. Artículos nacionales y extranjeros.

2. Investigaciones de corte empírico.

3. Publicaciones en revistas indexadas en alguna base de datos (Scopus, Scielo, Latindex u otra)

4. Actualidad, de 2016, 2017 o 2018.

5. Vínculos con la práctica docente. Suena una obviedad que un artículo se centra en la práctica docente, sin embargo, la realidad de la publicación científica es que algunos artículos son un tanto abstractos o son una reflexión teórica sobre una problemática. Los artículos acá seleccionados apuntan a situaciones más concretas, propias de la realidad de las aulas. El resumen de la información de los artículos se muestra en la Tabla 2. 
Tabla 2

Título del artículo y revista donde fueron publicados los artículos proporcionados a las personas participantes

\begin{tabular}{l|ll}
\hline Título del artículo & \multicolumn{2}{l}{ Revista donde fue publicado } \\
\hline $\begin{array}{l}\text { Acoso y ciberacoso en una muestra de alumnos de educación } \\
\text { secundaria (Martínez-Otero, 2017). }\end{array}$ & Revista de Profesorado (España) \\
\hline $\begin{array}{l}\text { Altas capacidades intelectuales y trastorno de } \\
\text { déficit de atención con hiperactividad: a propósito de un caso. } \\
\text { (Luque-Parra y Luque Rojas, 2017). }\end{array}$ & $\begin{array}{l}\text { Revista Perspectiva Educacional } \\
\text { (Chile) }\end{array}$ \\
\hline $\begin{array}{l}\text { Jefes de UTP nóveles en Chile: desafíos del } \\
\text { liderazgo pedagógico (Quiroga y Aravena, 2017) }\end{array}$ & $\begin{array}{l}\text { Revista Perspectiva Educacional } \\
\text { (Chile) }\end{array}$ \\
\hline $\begin{array}{l}\text { Aperturas y cierres para la inclusión educativa } \\
\text { de niños/as migrantes en Chile (Melis y Valderrama, 2018) }\end{array}$ & Estudios Pedagógicos (Chile) \\
\hline $\begin{array}{l}\text { Normas sociales, estereotipos, discriminación y violencia entre } \\
\text { pares (Machillot, 2017) }\end{array}$ & $\begin{array}{l}\text { Revista Mexicana de Investigación } \\
\text { Educativa (México) }\end{array}$ \\
\hline $\begin{array}{l}\text { La necesidad de trabajar los riesgos de internet en el aula } \\
\text { (Gamito, Aristizabal, Olasolo y Vizcarra, 2017). }\end{array}$ & Revista de Profesorado (España) \\
\hline $\begin{array}{l}\text { "Me duermo, pero igual aprendo": experiencias educativas } \\
\text { exitosas de jóvenes santafesinos de sectores bajos (Serra, }\end{array}$ & $\begin{array}{l}\text { Revista Páginas de Educación } \\
\text { (Uruguay) }\end{array}$ \\
$\begin{array}{l}\text { La17) violencia en las escuelas desde la perspectiva de sus } \\
\text { actores. El caso de una escuela secundaria de la Ciudad de } \\
\text { México (Cubas y Sarmiento, 2017) }\end{array}$ & Revista Educación (Costa Rica) \\
\hline $\begin{array}{l}\text { Liderazgo escolar y conflictos socioeducativos. Un estudio } \\
\text { exploratorio en liceos públicos chilenos (Villalobos, Peña, } \\
\text { Aguirre y Guerrero, 2018) }\end{array}$ & $\begin{array}{l}\text { Revista Calidad de la Educación } \\
\text { (Chile) }\end{array}$ \\
\hline
\end{tabular}

Fuente: Elaboración propia (2018).

Posterior a la entrega de los artículos, se les pidió que se reunieran en pequeños grupos para observar e indagar libremente los textos. Poco a poco, los fueron pasando de mano en mano con el propósito de que todos tuvieran la oportunidad de explorarlos. Después de la socialización de cada uno de ellos, se les pidió a los grupos que eligieran uno en particular y que lo leyeran con mayor detenimiento.

La utilización de los grupos focales buscaba el logro del objetivo número uno (Conocer las visiones que el alumnado de pedagogía tiene los artículos de investigación como un recurso pedagógico en su formación). Se realizaron cuatro grupos focales, de 9 a 10 participantes cada uno y tuvieron una duración aproximada de 45 minutos.

Las interrogantes generales planteadas en los grupos focales fueron establecidas previamente. Sin embargo, se asumía que muchas preguntas surgirían de acuerdo con el discurso de quienes participaron y con las interacciones entre ellos. Algunas de las interrogantes que guiaron la conversación fueron, por ejemplo: previo a esta actividad ¿qué contacto habías tenido con artículos de investigación?, ¿qué piensas sobre ellos en general?, ¿llamaron tu atención los textos proporcionados? 
Por su parte, la utilización de la entrevista-semiestructurada tuvo como propósito alcanzar el objetivo dos de este estudio (Conocer las visiones del alumnado de pedagogía sobre la vinculación entre los artículos de investigación y los elementos de su futura práctica docente). Después de que cada grupo leyera con mayor detenimiento el artículo seleccionado, se realizaron 15 entrevistas que tuvieron una duración aproximada de veinte minutos. Al tener un carácter semi-estructurado no se siguió una estructura rígida de organización, pero sí se pueden mencionar algunas de las preguntas realizadas:

- ¿Crees que este artículo se puede relacionar con elementos concretos de tu futura práctica?

- ¿Ves alguna relación entre el artículo que leíste y tu futuro rol como docente?

Cabe mencionar que tanto las entrevistas como los grupos focales se llevaron a cabo en dependencias de la Universidad de La Serena entre los meses de abril y mayo del año 2018. El periodo de análisis de datos se realizó entre los meses de junio y julio del mismo año.

\subsection{Procesamiento de análisis}

El análisis cualitativo de los datos fue abordado desde una perspectiva que recupera muchos de los principios de la Teoría Fundamentada (Glaser y Strauss, 1967), la cual propone una indagación por medio de fragmentos de información con significación propia. Posterior a la transcripción de las entrevistas y los grupos focales se determinaron las categorías que permitieron segmentar las citas más importantes. Se creó una unidad hermenéutica donde se determinaron una serie de códigos, que como ya hemos mencionado, surgieron tanto a priori como a posteriori a la recogida de la información. Posteriormente, se organizaron y jerarquizaron los códigos más importantes a través de familias semánticas y mapas conceptuales. En el transcurso de este trabajo, nos apoyamos en el programa Atlas.ti7, software que facilitó la segmentación, codificación y jerarquización de los datos. En la Figura 1 se muestran las relaciones entre los códigos de cada familia: 
Figura 1: Códigos y familias semánticas identificadas a partir de las entrevistas y grupos focales

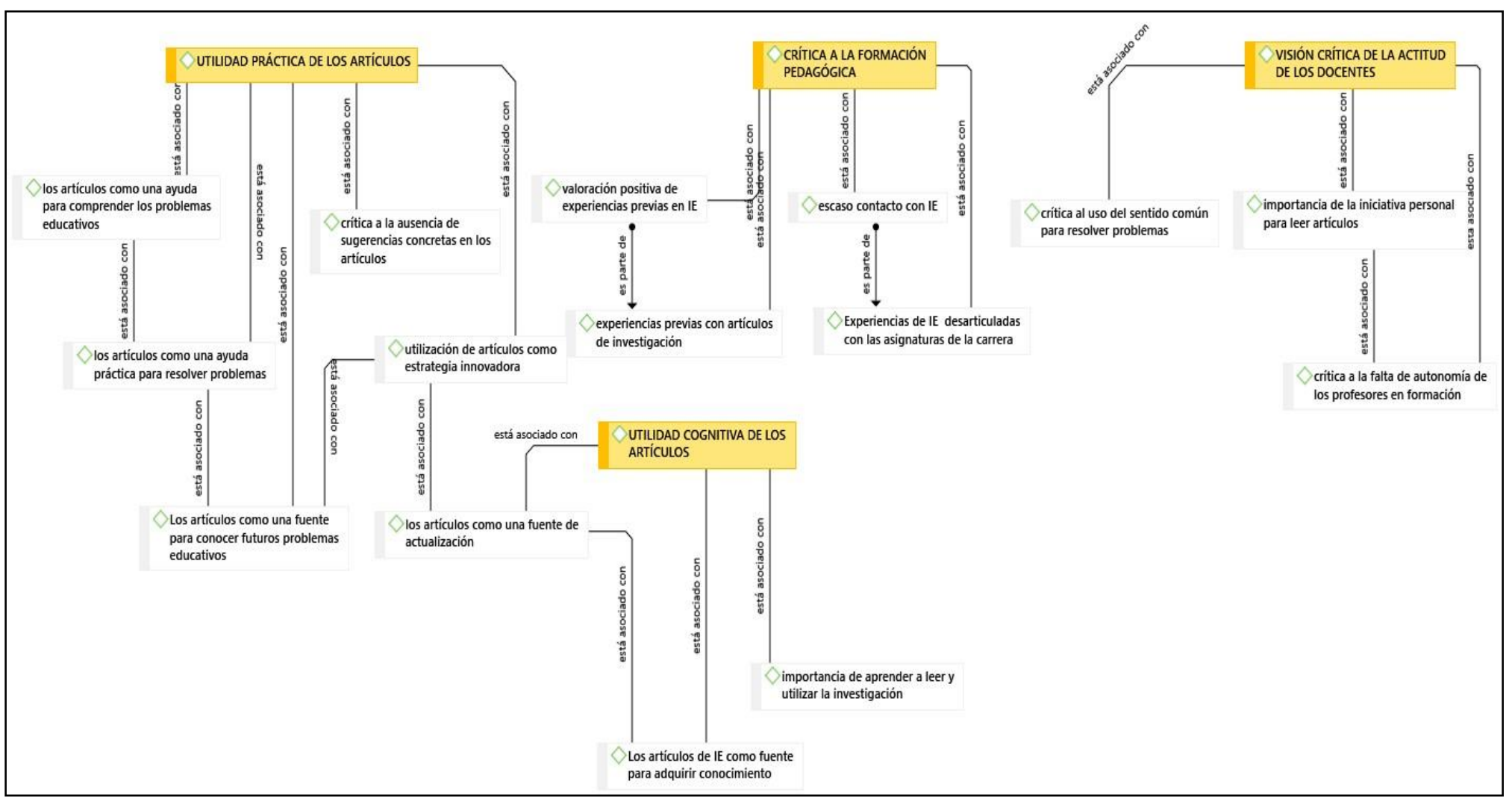

Fuente: Elaboración propia a partir del análisis de información en el software Atlas.ti.

\section{Resultados}

Los resultados del presente estudio se organizan según los objetivos planteados, el primero de ellos es conocer las valoraciones que el alumnado de pedagogía tiene de los artículos de investigación como un recurso pedagógico en su formación.

\subsection{Los artículos de investigación como una fuente para conocer, comprender y resolver los problemas educativos}

El alumnado participante, y sin mayores distinciones entre las carreras que estudian, percibe la investigación educativa (IE) como una herramienta que les permite conocer y comprender los problemas educativos, ya que les proporciona información importante para acercarse a diversos aspectos de la realidad educativa. Una de las participantes señala lo siguiente: 
La mayoría de los artículos que leemos son más cercanos a la realidad de lo que dicen ser. La mayoría aporta datos súper crudos, que no cualquiera se imagina, son de ayuda para ver estas situaciones desde otra perspectiva (grupo focal 4).

En este sentido, se menciona la importancia de poder relacionar la investigación con lo que sucede en la sala de clases, que los resultados de las investigaciones respondan a criterios prácticos, posibles de ser implementados en la escuela:

La investigación tiene que cumplir ese rol fundamental de ir conociendo el contexto en el cuál voy a trabajar (...), no creo que la investigación tenga que ser algo que se haga entre 4 paredes, por personas que ya llegaron a la cúspide en educación, sino que debe ser algo real que se conecte con la sala de clases (grupo focal 3).

Entre quienes participaron surge el interés de conocer, con mayor profundidad, los temas que se abordaron en los artículos, por ejemplo, en relación con el déficit atencional, con las necesidades educativas especiales o con el bullying. También mencionan que los textos presentan información concreta sobre estos tópicos, lo que les ayuda a comprender sus consecuencias y a reflexionar sobre sus soluciones:

Veo en los artículos algo súper concreto. El artículo nos dice: en el caso de 20 colegios se detectó bullying, se hicieron entrevistas y los resultados son tal y tal, y esto nos sirve para entender mejor la problemática (grupo focal 1).

Si bien las percepciones de quienes participaron dan cuenta de una alta valoración de los artículos leídos, también señalan que estos proporcionan una visión amplia de un problema educativo, que no necesariamente va a representar la realidad específica de una escuela. En los testimonios queda evidenciado que parte del estudiantado esperaría que los textos proporcionen soluciones más precisas a los problemas que le aquejan:

A mí me pasó que cuando leí los artículos vi que hablaban del tema en general, pero no daban una solución como concreta, buscaba eso en las conclusiones, pero no lo encontraba. Era interesante, pero no daban algo que uno pudiera aplicar, una ayuda real (grupo focal 2). 


\subsection{Los artículos de investigación como una herramienta para adquirir nuevos conocimientos y actualizarlos}

Cabe mencionar que, en este apartado y en los siguientes, no se identifican diferencias importantes en las valoraciones del estudiantado de acuerdo con la carrera que estudia. El alumnado de pedagogía, en general, expresa que la lectura de los artículos de investigación les permitió conocer nuevos aspectos relativos a la enseñanza, al aprendizaje y a diversas problemáticas actuales que enfrenta la educación. Valoran positivamente que, a través de la revisión de los textos, hayan accedido a nuevos saberes y conceptos pertinentes al contexto del aula. Uno de ellos dice lo siguiente:

A mí me llamó la atención la variedad de temas que se pueden tocar en los artículos (...) que con un par de artículos uno puede hacerse una idea sobre cómo tratar los problemas. En todos los artículos se puede encontrar algo valioso, por ejemplo, para enseñar mejor, para trabajar el acoso escolar, el bullying, que son cosas que uno no aprende en cualquier parte, pero con la lectura de estos artículos uno puede aprender y aplicar (grupo focal 1).

Algunas de las personas participantes son enfáticas al decir que la lectura de artículos facilita el perfeccionamiento y la actualización de los conocimientos necesarios para contar con un respaldo pedagógico en el aula. Además, enfatizan que el profesorado no puede quedarse estancado en las prácticas y contenidos que se enseñaban en el pasado y que debe haber una voluntad personal para renovar los saberes que ya se poseen:

No nos podemos quedar con las prácticas de hace 50 o 100 años (...) por eso la investigación y los artículos son importantes, ayudan a tener las herramientas base del profesor para poder afrontar el aula, cómo se van desenvolviendo los niños en comparación a lo que ocurría en el pasado (grupo focal 2).

Parte del estudiantado destaca, además, que no se trata solo de actualizar los propios saberes, sino que se debe hacer un esfuerzo por ayudar a las demás personas docentes a que también actualicen sus conocimientos:

Nuestro rol también es explicar e informar a los demás docentes dentro de un establecimiento educacional lo que hemos aprendido en los artículos, por ejemplo, las características que poseen los niños con altas capacidades intelectuales, trastorno de déficit atencional u otras cosas relevantes (grupo focal 3). 
Además de adquirir y actualizar los conocimientos, el alumnado en formación señala que los contenidos de los artículos de investigación pueden reemplazar las carencias que tienen en su formación pedagógica. Sienten que leyendo este tipo de textos pueden suplir todos aquellos aspectos que quedan pendientes de abordar durante los cuatro años y medio de la carrera:

Siempre en la formación vamos a encontrar cosas que no nos enseñan, por ejemplo, inclusión, bullying, ni aunque la carrera durara 7 años, siempre van a ver (sic) cosas que quedarán pendientes, cosas que nos falten (...) Si uno sabe buscar, lee un artículo, y ahí tiene una forma de tratar un problema (grupo focal 1)

\subsection{Valoración de las experiencias previas en temas de investigación educativa}

Es importante mencionar que, en general, el estudiantado participante valora positivamente las experiencias que han tenido con la investigación educativa, como, por ejemplo, el análisis de investigaciones recientes o el diálogo con personas que investigan. También destacan favorablemente las posibilidades que han tenido de trabajar con artículos de investigación a través de la búsqueda de artículos de calidad, o por medio de la lectura de estos durante sus clases. Incluso, algunos de ellos afirman que buscan y leen artículos regularmente:

(...) A nosotras las profesoras nos han enseñado que busquemos información en revistas científicas. Cuando tengo que estudiar algo me voy directo a las revistas en Scielo por ejemplo y ahí busco información (grupo focal 4).

Por ejemplo, con la profe de teoría, también vimos paper, eso fue en segundo, en el último semestre, eso nos ayudó a hacernos una imagen del aula, veíamos el contexto escolar con más claridad (grupo focal 1 ).

La reflexión de este estudiantado refleja que el hábito de leer artículos se debe, en parte, a la estimulación y motivación que transmiten personas académicas que han participado en su formación. A pesar de esta valoración positiva de las experiencias vividas, también hay estudiantes que manifiestan un descontento con la escasa formación en investigación que han recibido. Si bien estas experiencias les han parecido interesantes, creen que son insuficientes: 
Si nosotros no aprendemos a investigar ahora en la formación inicial difícilmente vamos a salir de acá y diremos: tengo un problema con este curso y voy a investigar, porque quizás ni siquiera sepa dónde buscar (grupo focal 4).

Una de las principales críticas realizadas por el estudiantado es que las experiencias en investigación educativa que han tenido hasta el momento han sido más cercanas a perspectivas teóricas, que usualmente están separadas de aspectos prácticos. Además, mencionan que la IE debe ser trabajada de manera progresiva y con mayor cobertura, a fin de evitar que su escasa presencia y desarticulación entre las asignaturas aumente la dificultad de su comprensión. Cuando esto ocurre, son asignaturas específicas de altas exigencias y habilidades de investigación que generan sorpresa por el alto grado de complejidad que demandan:

Quizás falta un poco eso, el conectar esa asignatura con todo el desarrollo de la carrera, el primer semestre ir conociendo los artículos en otras asignaturas, trabajar en base a ellos, para ya tener una idea de cómo son y no llegar a la asignatura y sorprenderse y tener que hacer una investigación de la nada" (grupo focal 3).

Ante ello, el estudiantado manifiesta que la formación inicial docente es la etapa donde se debiesen acercar a la investigación educativa, ya que las habilidades que se desarrollen les permitirán contribuir a un proceso de mejora en su labor pedagógica. Un aspecto importante es que el alumnado participante considera que los artículos de investigación son una herramienta pedagógica efectiva, ya que les permiten distanciarse de una clase tradicional, que usualmente está centrada en aspectos teóricos.

Es relevante mencionar las valoraciones del estudiantado de la carrera de Educación Diferencial, quienes manifiestan que la investigación educativa sí forma parte importante de su formación docente:

Como carrera de diferencial, en Teoría de las dificultades tuvimos que realizar una revisión bibliográfica del trastorno que le tocó a cada uno (...) tuvimos que buscar libros y artículos científicos y de ahí sacar información para agregarla a nuestra monografía (grupo focal 3)

Antes de pasar a los hallazgos relacionados con el segundo objetivo, cabe mencionar que algunas de las personas participantes se muestran críticas con el colectivo docente, 
cuando indican que parte del profesorado recurre a la opinión de los pares o al sentido común para resolver los problemas educativos, en desmedro de la utilización de artículos de investigación. Para ellos, el contenido de los artículos les puede proporcionar una información comprobada, que ha sido construida con datos empíricos. Estas características les dan a los textos un "peso" académico que debería ser considerado por el cuerpo docente al momento de buscar ayuda para enfrentar las coyunturas del aula:

Es un error que se comete que cuando tengo un problema le consulto a otro profesor, el que probablemente me responda desde el sentido común y sin ninguna otra base y sin yo poder, por ejemplo, en vez de preguntarle a él, buscar por mis propios medios un artículo y poder después utilizarlo y sacar más provecho, porque mi par estará exactamente en las mismas condiciones que yo (grupo focal 1).

Otro de los aspectos a destacar entre las opiniones del alumnado participante es que algunos de ellos entregan sugerencias sobre cómo debería ser su formación en investigación. Mencionan ciertas acciones o iniciativas que se pueden llevar a cabo para conocer la investigación educativa y también los artículos de investigación:

Si leemos artículos en pareja podemos debatir sobre un tema y otra pareja hace lo mismo y así sucesivamente, entonces en voz alta podemos aprender todos de todos los temas (grupo focal 4).

Hacer, quizás, una investigación dentro de nuestra carrera, con profesionales egresados de nuestra carrera, con nuestra misma malla, porque no sirve de nada hacerlo con la nueva malla, cómo están en el aula, cómo se están enfrentado. Si realmente están preparados, con algún tipo de investigación que diga si estamos realmente preparados o no (grupo focal 3).

El segundo objetivo de este estudio es conocer las visiones que el alumnado tiene sobre la vinculación entre los artículos de investigación y los elementos de su futura práctica docente. En términos generales, quienes participaron en las entrevistas sienten que el contenido de los artículos sí tiene vinculación directa con su futura práctica docente. Lo que más destacan de esta vinculación es que la lectura de los artículos les ha permitido la visibilización concreta de los problemas educativos a los que se enfrentarán en el futuro y la vinculación del contenido de los artículos con su rol como profesorado jefe. 
Por ejemplo, el artículo Acoso y ciber acoso en una muestra de alumnos de educación secundaria generó en algunos casos un debate sobre cómo ha aumentado el uso nocivo del internet del estudiantado y sobre la importancia de que el profesorado tome conciencia de un problema cada vez más alarmante dentro de las aulas. Los futuros profesores y profesoras destacan que infantes y jóvenes manejan perfectamente la tecnología y que van varios pasos más adelantados que los padres, madres, incluso, que el propio profesorado, por lo que la lectura de artículos actuales podría contribuir a estar más y mejores informados al respecto:

Para tratar un tema tan delicado como el ciberacoso se requiere de conocimientos, no todo es por sentido común, especialmente con el tema del internet. Los estudiantes van por delante de los profesores y de los padres en estos temas, entonces debemos ser capaces de detenernos a leer sobre los estudios que ya existen y así estar bien informados sobre la realidad que vamos a enfrentar en poco tiempo más (entrevista 6).

Algo similar ocurrió con el artículo Aperturas y cierres para la inclusión educativa de niños/as migrantes en Chile. Respecto a este texto, las personas participantes señalan que la diversidad étnica que hay en las escuelas actualmente no es la misma que conocieron hace unos pocos años cuando eran estudiantes. Por ello, reconocen que como futuro profesorado deben asumir estos cambios desde la formación, para adoptar, oportunamente, una postura inclusiva y reflexiva sobre la llegada de estudiantes inmigrantes:

En los últimos años en Chile ha crecido el porcentaje de inmigración y por eso ha aumentado la presencia de niños migrantes en los colegios. Por esto se hace necesario que nosotras como futuras profesoras adoptemos una postura a este respecto que empatice con las distintas realidades de los niños (entrevista 11).

Respecto a la vinculación específica del contenido de los artículos con la realidad del aula, el alumnado destaca, principalmente, que esta conexión la observan con mayor claridad en el rol del profesorado jefe, que deberán asumir cuando ingresen al mundo laboral. En beneficio de la comprensión de este artículo, por parte de todos los lectores, es importante especificar que en Chile el profesorado jefe (profesor tutor, preceptor, profesor guía o lead teacher en otros países) es una figura casi inherente a la de ser docente de asignatura y, en la mayoría de los casos, asumir la jefatura de un curso es parte de tu contrato laboral. El profesorado jefe tiene múltiples responsabilidades, por lo que se 
convierte en una pieza clave en el proceso de enseñanza-aprendizaje del grupo de infantes, en el compromiso de sus padres, en la cooperación del equipo técnico y en la administración de la escuela (Vélez, Schiefelbein y Valenzuela, 1994).

Quienes participan en este estudio, tal vez recordando lo que sucedía cuando eran estudiantes de colegio, están conscientes de la complejidad del rol de profesorado jefe. Por ello, ven en la lectura de los artículos una valiosa forma de adquirir conocimientos y herramientas que les permitan desenvolverse mejor en esta función:

Ser profesor jefe es muy complejo, da la sensación de que el profe jefe debe ser un psicólogo, amigo, educador, etc. y la verdad es que no sé si estamos preparados para ellos (...) es bueno leer este tipo de cosas para aprender sobre situaciones educativas que tendremos que enfrentar (entrevista 2).

También mencionan la lamentable realidad del bullying, el ciberbullying, la discriminación, las discusiones que acaban en violencia, etc. y la importancia de que el profesorado jefe conozca información actualizada sobre la forma más adecuada de abordar estos temas con los niños y niñas, y también con sus padres y madres:

Con los padres y madres también hay que hacer un trabajo con estos temas porque a veces ellos ignoran cómo sus hijos usan el internet, sería bueno que en las reuniones se les comentaran resultados reales de artículos vigentes (cita extraída de las entrevistas).

\section{Conclusiones}

En términos generales, el presente estudio confirma las aportaciones de trabajos anteriores que sugieren que la investigación educativa es relevante y fundamental para el profesorado como personas informadas, críticas y reflexivas (Haberfellner y Fenzl, 2017). En coincidencia con algunos estudios (Damşa, 2018; Demircioglu, 2008; Jyrhämä et al., 2008), el futuro profesorado ve en la investigación una forma de conocer nuevos elementos relativos a la enseñanza y al aprendizaje.

Por otra parte, y en consonancia con el trabajo de Jyrhämä et al. (2008) y Perines y Murillo (2017b), el alumnado de las pedagogías considera que las experiencias en investigación y en lectura de artículos que han tenido hasta el momento, han significado un aporte para su preparación profesional. Sin embargo, critican la poca conexión entre las 
asignaturas relativas a la investigación y la poca formación intencionada en esta materia, lo que coincide con lo señalado por Gitlin et al. (1999) y MacDonald et al. (2001).

Este trabajo también encontró que, para el alumnado de pedagogía, la revisión de artículos contribuye a conocer, comprender y resolver los problemas educativos, aunque con algunos matices. Hay opiniones que critican la ausencia de soluciones concretas en los artículos, lo que coincide con lo señalado por Hammersley (2004) cuando destaca el eterno conflicto entre generalizar los conocimientos que surgen de la investigación y la necesidad de encontrar soluciones específicas para un problema práctico. Esta dicotomía ha permanecido en el tiempo y representa un punto de inflexión fundamental para las personas investigadoras, y al mismo tiempo, para el profesorado y las escuelas. Por otra parte, y de forma similar a los hallazgos de Murillo et al. (2017) y Pendry y Husbands (2000), el estudiantado de pedagogía tiene una visión positiva de los artículos, pero reconocen que su lectura sigue siendo limitada.

En coincidencia con los resultados de Zeuli (1994) y Bartels (2003), los hallazgos de este artículo expresan que el profesorado del futuro tiene una mejor valoración de los artículos cuando estos tienen conexión con la práctica y destacan que la lectura de los artículos les ayudó a tener una visión más "real" de los conflictos que enfrentarán. Más allá de esta coincidencia, resulta interesante que el presente artículo aborda la lectura de artículos desde la formación inicial, lo que amerita una reflexión desde las bases de la preparación profesional de las personas docentes.

A partir de los resultados del presente artículo surgen sugerencias para que las personas formadoras de docentes, las administraciones de las universidades, las personas investigadoras educativas y quienes se encargan del área de investigación de las universidades tomen conciencia de la importancia de la formación en investigación del futuro profesorado y de la inclusión oportuna de los artículos de investigación. Revisemos las sugerencias que, a partir de los resultados del presente artículo, surgen para cada uno de estos estamentos:

Personas formadoras de docentes: Quienes tienen la responsabilidad de educar al profesorado del futuro: 1. Deben promover que las acciones de investigación formen parte activa de sus metodologías de aula. Es cierto que cada docente intenta cumplir con su programa, sin embargo, la inclusión de la investigación educativa y de los artículos de investigación puede concretarse de manera transversal a las asignaturas. Las sugerencias de quienes participaron en este estudio pueden servir como referencia. 2. Se pueden 
realizar periódicamente lecturas grupales de artículos, debates sobre resultados de estudios o participación del estudiantado en pequeñas investigaciones. 3. Al respecto sería interesante que los formadores incluyeran, entre sus metodologías, aspectos del "Aprendizaje Basado en la Investigación" (ABI) (En inglés, inquiry based learning-IBL), que Peñaherrera, Chiluiza y Ortiz (2014, p. 8) definen como una "estrategia didáctica activa centrada en el alumno, para desarrollar competencias que le permitan realizar una investigación".

Administración de las universidades: En las valoraciones del alumnado subyace: 1. Una crítica a la formación pedagógica que han recibido porque sienten que ha sido demasiado teórica y descontextualizada de la realidad escolar. Al leer los artículos se dan cuenta de que no cuentan con herramientas tangibles para resolver situaciones que pueden surgir en el aula. 2. Este escenario debe suscitar cambios en los programas y una revisión exhaustiva de las asignaturas propias de la pedagogía. 3. Asimismo, las administraciones deben tener la autocrítica necesaria para construir nuevos caminos en la formación de docentes en lo que cobra una especial relevancia la opinión del estudiantado.

Personas investigadoras educativas: El desafío de esas personas es aún mayor porque: 1. Lo que está pidiendo el alumnado de las pedagogías es más investigación vinculada a la práctica, que no siempre es la prioridad de quienes investigan. Estas últimos deben contar con protocolos que exijan la construcción de investigaciones basadas en la realidad educativa. 2. El investigador o investigadora no debe renunciar a sus propios intereses (la anhelada publicación de artículos en Wos, por ejemplo), pero sí debe estipular en su planificación científica la inclusión de estudios que conecten la teoría y la práctica de manera más precisa.

Personas encargadas del área de investigación de las universidades: Para lograr una mejor formación en investigación es fundamental que: 1. Las futuras personas docentes tengan experiencias como "pequeños" investigadores o investigadoras, que, por supuesto, deben ser acordes a la etapa de formación en la que se encuentran. Es el mismo estudiantado quien menciona esta idea cuando indica que les gustaría participar en investigaciones donde tengan la posibilidad de interactuar con otros grupos de estudiantes de pedagogía, con docentes en ejercicio, con los niños y niñas de las escuelas y con quienes investigan. 2. Para que esto se materialice quienes lideran las agendas de investigación de las universidades deben estipularlo en sus lineamientos de acción. Un buen ejemplo de ello son los concursos de Investigación Pedagógica Aplicada (IPA) que se están llevando a cabo 
en la Universidad de La Serena desde el año 2016. Estos proyectos buscan la realización de investigaciones que surjan desde problemáticas de las escuelas y que involucren a los actores del sistema escolar con la colaboración de estudiantes de pedagogía y personas dedicadas a la investigación.

Las limitaciones del presente estudio son, por una parte, abordar las valoraciones de estudiantes de solo una universidad y, por otra, utilizar la metodología cualitativa de forma exclusiva. Tal vez, los futuros trabajos en torno a este tema podrían abarcar valoraciones del alumnado de pedagogía de otras universidades a través de un enfoque metodológico mixto que incluya metodología cualitativa y cuantitativa. Esto permitiría profundizar en los resultados acá obtenidos y enriquecer el debate ya instalado.

La utilización de la investigación y la inclusión de artículos de investigación dentro de las pedagogías es urgente. Como sistema educativo necesitamos de un profesorado mejor preparado, que razone, que cuestione, que se informe, que esté actualizado y que sea capaz de reflexionar sobre la práctica. Este tipo de docentes es lo que merecen nuestros niños y nuestras niñas.

\section{Agradecimientos}

Se agradece al estudiantado de las pedagogías de la Universidad de La Serena (Chile), por su participación voluntaria en esta investigación.

\section{Referencias}

Alhumidi, Hamed y Uba, Sani Yantandu. (2017). Arabic Language Teachers' Engagement with Published Educational Research in Kuwait's Secondary Schools. International Journal of Higher Education, 6(2), 20. Recuperado de https://eric.ed.gov/?id=EJ1134452.

Altrichter, Herbert y Mayr, Johannes. (2004). Forschung in der Lehrerbildung. En Sigrid Blömke, Peter Reinhold, Gerhard Tulodziecki y Johannes Wildt (Eds.), Handbuch Lehrerbildung (pp. 160-184). Bad Heilbrunn., Germany: Klinkhardt.

Bartels, Nat. (2003). How teachers and researchers read academic articles. Teaching and Teacher Education, 19(7), 737-753. Recuperado de https://www.sciencedirect.com/science/article/pii/S0742051X03000799.

Corbin, Juliet and Strauss, Anselm. (2008). Basics of qualitative research. Technics and procedures for developing grounded theory. Londres: Sage Publications. Recuperado de

https://www.researchgate.net/publication/277197202 Basics of Qualitative Research Techniques and Procedures for Developing Grounded Theory 
Counsell, Christine, Evans, Michael, McIntyre, Donald y Raffan, John. (2000). The usefulness of educational research for trainee teachers' learning. Oxford Review of Education, 26(3-4), 467-482. Recuperado de https://www.jstor.org/stable/1050771?seq=1\#metadata info tab contents

Damşa, Crina (2018). Research and Development Tasks in Teacher Education: Institutional Framing and Student Experiences. In Peter Maassen, Monika Nerland and Lyn yates (eds.), Reconfiguring Knowledge in Higher Education (Higher Education Dynamics 50, pp. 149-167). Springer, Cham. Recuperado de https://link.springer.com/chapter/10.1007/978-3-319-72832-2 9.

Demircioglu, Ismael. (2008). Learning How to Conduct Educational Research in Teacher Education: A Turkish Perspective. Australian Journal of Teacher Education, 33(1), 1-17. Recuperado de https://files.eric.ed.gov/fulltext/ED504400.pdf

Eccles, Jacquelynne y Wigfield, Allan (2002). Motivational beliefs, values, and goals. Annual review of psychology, 53, 109-132. Recuperado de https://www.researchgate.net/publication/281345525 Motivational Beliefs Values and Goals .

Elliott, John (2001). Making evidence-based practice educational. British Educational Research Journal, 27(5), 555-574. Recuperado de https://onlinelibrary.wiley.com/doi/abs/10.1080/01411920120095735.

Forner, Angel (2000). Investigación educativa y formación del profesorado. Revista Interuniversitaria de Formación del Profesorado, (39), 33-50. Recuperado de http://w.aufop.com/aufop/uploaded files/articulos/1223509365.pdf.

Gil Álvarez, José Luis., León González, Jorge Luis., y Morales Cruz, Mabel. (2017). Los paradigmas de investigación educativa, desde una perspectiva crítica. Revista Conrado, 13(58), 72-74. Recuperado de http://conrado.ucf.edu.cu/index.php/Conrado

Gitlin, Andrew., Barlow, Linda., Burbank, Mary., Kauchak, Don. y Stevens, Tracy. (1999). Preservice teachers' thinking on research: Implications for inquiry oriented teacher education. Teaching and Teacher Education, 15(7), 753-769. Recuperado de https://pdfs.semanticscholar.org/d34e/31b3e09955831a4e6991a7d5c65769d54f28.pdf.

Glaser, Barney y Strauss Aldine (1967) The Discovery of Grounded Theory: Strategies For Qualitative Research. New York NY: Aldine de Gruyter. Recuperado de http://www.sxf.uevora.pt/wp-content/uploads/2013/03/Glaser 1967.pdf.

Grossman, Pam y McDonald, Morva. (2008). Back to the future: Directions for research in teaching and teacher education. American Educational Research Journal, 45(1), 184205. Recuperado de https://journals.sagepub.com/doi/10.3102/0002831207312906

Haberfellner, Christina. and Fenzl, Thomas. (2017). The utility value of research evidence for educational practice from the perspective of preservice student teachers in Austria- $A$ qualitative exploratory study. Journal for Educational Research Online, 9(2), 69-87. Recuperado de https://www.pedocs.de/volltexte/2017/14897/pdf/JERO 20172 Haberfellner Fenzl Th e utility value.pdf. 
Hammersley, Martyn (2004). Action research: a contradiction in terms? Oxford Review of Education, 30(2), 165-181. Recuperado de http://www.leeds.ac.uk/educol/documents/00002130.htm.

Jyrhämä, Riitta., Kynäslahti, Heikki., Krokfors, Leena., Byman, Reijo, Maaranen, Katriina, Toom, Auli. y Kansanen, Pertti. (2008). The appreciation and realization of research-based teacher education: Finnish students' experiences of teacher education. European Journal of Teacher Education, 31(1), 1-16. Recuperado de https://www.tandfonline.com/doi/abs/10.1080/02619760701844993.

Kutlay, Nesrin. (2013). A Survey of English Language Teachers' Views of Research. Procedia-Social and Behavioral Sciences, 70, 188-206. Recuperado de https://www.sciencedirect.com/science/article/pii/S1877042813000566.

MacDonald, Malcolm., Badger, Richard. y White, Goodith. (2001). Changing values: what use are theories of language learning and teaching? Teaching and Teacher Education, 17(8), 949-963. Recuperado de https://www.sciencedirect.com/science/article/pii/S0742051X01000427

Murillo, Javier, Perines, Haylen y Lomba, Lucía. (2017). La comunicación de la investigación educativa. Una aproximación a la relación entre la investigación, su difusión y la práctica docente. Profesorado, Revista de Currículum y Formación del Profesorado, 21(3), 183-200. Recuperado de https://www.redalyc.org/pdf/567/56752489009.pdf.

Pendry, Anna y Husbands, Chris. (2000). Research and practice in history teacher education. Cambridge Journal of Education, 30(3), 321-334. Recuperado de https://www.tandfonline.com/doi/abs/10.1080/03797720600625275.

Peñaherrera, Mónica., Chiluiza, Katherine. y Ortiz Colón, Ana. (2014). Inclusión del Aprendizaje Basado en Investigación ( $\mathrm{ABI}$ ) como práctica pedagógica en el diseño de programas de postgrados en Ecuador. Elaboración de una propuesta. Journal for Educators, Teachers and Trainers, 5(2), 204-220. Recuperado de https://www.ugr.es/ jett/pdf/Vol5(2) 015 jett Penaherrera Chiluiza Ortiz.pdf.

Perines, Haylen y Murillo Javier. (2017a). Percepciones de los docentes en formación sobre la investigación educativa. Estudios Pedagógicos, 43(1), 251-268. Recuperado de https://scielo.conicyt.cl/scielo.php?pid=S0718$07052017000100015 \&$ script=sci arttext\&tlng=e.

Perines, Haylen y Murillo, Javier. (2017b). ¿Cómo mejorar la investigación educativa? Sugerencias de los docentes. Revista de la Educación Superior, 46(181), 89-104. Recuperado de https://www.sciencedirect.com/science/article/pii/S0185276017300055.

Pring, Richard. (2017). Research and the Undermining of Teacher Education. In Michael A. Peters, Bronwen Cowie and lan Menter (eds.), A companion to Research in Teacher $\begin{array}{llll}\text { Education (pp. 609-620). Recuperado de } & \end{array}$ https://link.springer.com/content/pdf/10.1007\%2F978-981-10-4075-7.pdf 
Slavin, Robert. (2002). Evidence-based education policies: Transforming educational practice and research. Educational Researcher, 31(7), 15-21. Recuperado de https://journals.sagepub.com/doi/abs/10.3102/0013189X031007015.

Vélez, Eduardo., Schiefelbein, Ernesto. y Valenzuela, Jorge. (1994). Factores que afectan el rendimiento académico en la educación primaria: Revisión de la literatura de América $\begin{array}{lllll}\text { Latina } & y & \text { el }\end{array}$ http://repositorio.minedu.gob.pe/handle/123456789/4317.

Vetter, Peter. and Ingrisani, Daniel. (2013) The Benefi ts Pre-service Teachers Derive from Tuition in Research Methodology. Beiträge zur Lehrerinnen- und Lehrerbildung, 31(3), 321-332. Recuperado https://www.pedocs.de/volltexte/2017/13853/pdf/BZL 20133321 332.pdf.

Zeuli, John. (1994). How do teachers understand research when they read it? Teaching and Teacher Education, 10(1), 39-55. Recuperado de https://www.sciencedirect.com/science/article/pii/0742051X94900396. 
Revista indizada en

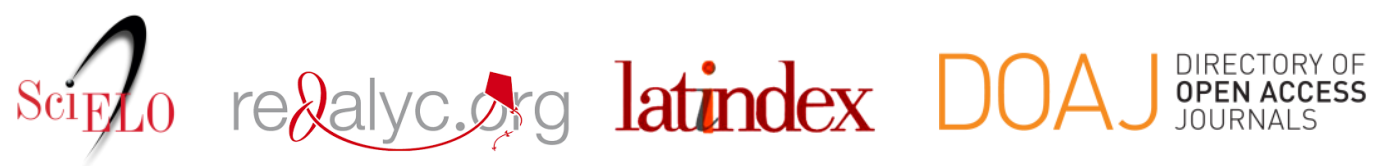

Distribuida en las bases de datos:

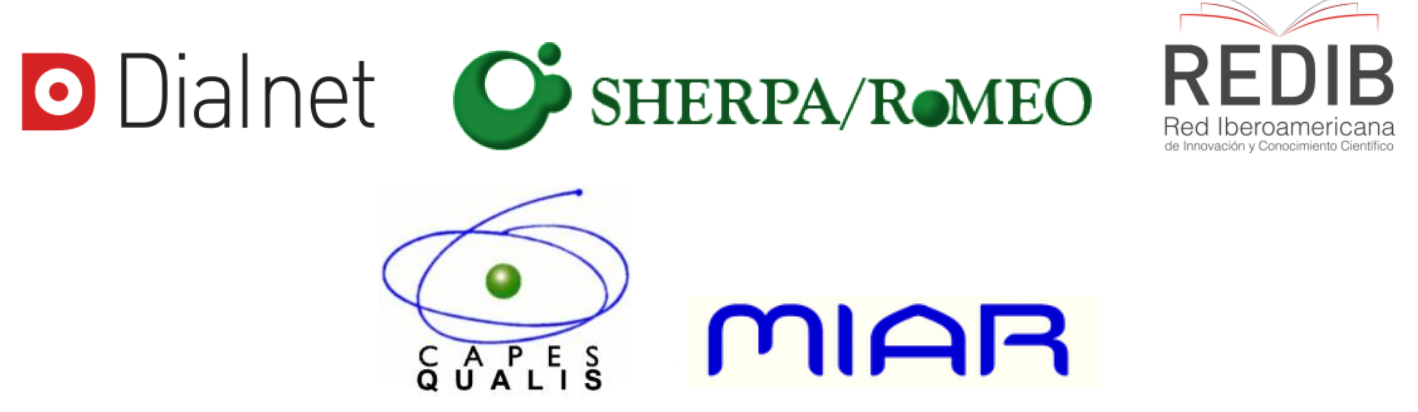

